\title{
Determinants of empirical antipseudomonal antibiotic prescription for adults with pneumonia in the emergency department
}

Nuria Angrill', Miguel Gallego ${ }^{1,2^{*}}$ (D), Juli Font ${ }^{3}$, Jordi Vallés ${ }^{2,4}$, Anisi Morón ${ }^{5}$, Eduard Monsó ${ }^{1,2}$ and Jordi Rello $2,6,7$

\begin{abstract}
Background: Antipseudomonal antibiotics should be restricted to patients at risk of Pseudomonas aeruginosa infection. However, the indications in different guidelines on community-acquired pneumonia (CAP) are discordant. Our objectives were to assess the prevalence of antipseudomonal antibiotic prescriptions and to identify determinants of empirical antibiotic choices in the emergency department.
\end{abstract}

Methods: Observational, retrospective, one-year cohort study in hospitalized adults with pneumonia. Antibiotic choices and clinical and demographic data were recorded on a standardized form. Antibiotics with antipseudomonal activity were classified into two groups: a) $\beta$-lactam antipseudomonals ( $\beta$-APS), including carbapenems, piperacillin / tazobactam or cefepime (in monotherapy or combination) and b) monotherapy with antipseudomonal quinolones.

Results: Data were recorded from 549 adults with pneumonia, with Pseudomonas aeruginosa being isolated in only nine (1.6\%). Most (85\%) prescriptions were compliant with SEPAR guidelines and 207 (37\%) patients received antibiotics with antipseudomonal activity (14\% $\beta$-APS and 23\% levofloxacin). The use of $\beta$-APS was independently associated with ICU admission (OR 8.16 95\% Cl 3.69-18.06) and prior hospitalization (OR 6.76 95\% Cl 3.02-15.15), while levofloxacin was associated with negative pneumococcal urine antigen tests (OR 3.41 95\% Cl 1.70-6.85) but negatively associated with ICU admission (OR $0.2695 \% \mathrm{Cl} 0.08-0.86$ ). None of these factors were associated with $P$ aeruginosa episodes. In univariate analysis, prior $P$ aeruginosa infection/colonization ( $2 / 9$ vs 6/372, $p=0.013$ ), severe COPD (3/9 vs 26/372, $p=0.024$ ), multilobar involvement (7/9 vs $119 / 372, p=0.007)$ and prior antibiotic (6/9 vs 109/372, $p=0.025)$ were significantly associated with $P$ aeruginosa episodes.

Conclusions: Antipseudomonal prescriptions were common, in spite of the very low incidence of Pseudomonas aeruginosa. The rationale for prescription was influenced by both severity-of-illness and pneumococcal urine antigen test (levofloxacin) and prior hospitalization and ICU admission ( $\beta$-APS). However, these factors were not associated with $P$ aeruginosa episodes. Only prior $P$ aeruginosa infection/colonization and severe COPD seem to be reliable indicators in clinical practice.

Keywords: Pneumonia, P aeruginosa, Antipseudomonal antibiotics, Levofloxacin, Guidelines

\footnotetext{
* Correspondence: mgallego@tauli.cat

'Department of Respiratory Medicine, Parc Taulí Hospital Universitari, Institut

d'Investigació i Innovació Parc Taulí I3PT, Universitat Autònoma de Barcelona, Sabadell, Spain

${ }^{2}$ CIBER de Enfermedades Respiratorias (CIBERES), Instituto de Salud Carlos III, Madrid, Spain

Full list of author information is available at the end of the article
}

(c) The Author(s). 2020 Open Access This article is licensed under a Creative Commons Attribution 4.0 International License, which permits use, sharing, adaptation, distribution and reproduction in any medium or format, as long as you give appropriate credit to the original author(s) and the source, provide a link to the Creative Commons licence, and indicate if changes were made. The images or other third party material in this article are included in the article's Creative Commons licence, unless indicated otherwise in a credit line to the material. If material is not included in the article's Creative Commons licence and your intended use is not permitted by statutory regulation or exceeds the permitted use, you will need to obtain permission directly from the copyright holder. To view a copy of this licence, visit http://creativecommons.org/licenses/by/4.0/. The Creative Commons Public Domain Dedication waiver (http://creativecommons.org/publicdomain/zero/1.0/) applies to the data made available in this article, unless otherwise stated in a credit line to the data. 


\section{Background}

The clinical practice guidelines recommend that antimicrobial treatment for patients with community-pneumonia (CAP) should be prescribed empirically after evaluation of severity at presentation and pre-existing comorbid disease, and should be stratified based on prognostic risk scores [1-4]. However, the recommendations are inconsistent and compliance is low; the choice of antibiotic treatment at the bedside is a complex process influenced by several factors that have not yet been definitively characterized. Factors proposed in the literature include previous hospitalization, previous antibiotic exposure and underlying diseases, but there are others that are dependent on hospital organization, local patterns of resistance, background speciality and cost-effectiveness [5-7].

Compliance of antibiotic treatment with the guidelines has been associated with improved outcomes, but mortality rates rise in patients at risk of multi-resistant microorganisms such as $P$ aeruginosa $[8,9]$. However, antipseudomonal antibiotics are considered as broad spectrum and, due to their potential for causing collateral damage $[10,11]$, their administration should be restricted.

Pneumonia due to $P$ aeruginosa occurs in several distinct syndromes such as community-acquired pneumonia (CAP) and bacteremia in neutropenic hosts, and in intubated patients [12] In CAP, coverage of $P$ aeruginosa is controversial due to the different rates of prevalence reported in the literature [1318]. Factors such as structural lung diseases (especially bronchiectasis), repeated exacerbations of severe chronic obstructive pulmonary disease (COPD), chronic oral steroid administration, alcoholism and frequent ( $>4$ courses per year) or recent antibiotic therapy have been associated with $P$ aeruginosa isolates [1, 2, 4, 19]. However, a large retrospective study reported that a significant proportion of patients with CAP due to $P$ aeruginosa did not present any of these conditions [17]. Although not routinely included in most CAP guidelines, a certain degree of immunocompromise is frequent in patients with community-acquired pneumonia, with neutropenia being a common comorbid condition [20].

The aim of this study was to assess the prevalence of the use of antibiotics with antipseudomonal activity and the determinants of empirical choice, in patients attended for pneumonia in the emergency department. We hypothesizied was that antipseudomonal antibiotics might be prescribed more frequently than needed, and that determinants of prescription differ between quinolones and other choices.

\section{Methods}

Retrospective, observational cohort study of all consecutive patients attended in the emergency department (ED) at Parc Tauli Hospital (682 beds and a reference population of 391,460 inhabitants), between September 2010 and September 2011. The protocol was approved by the Sabadell Hospital Ethics Committee (2014/538).

\section{Study population}

All patients $\geq 18$ were evaluated for study inclusion. Patients were identified by primary ICD-9 codes for pneumonia (480.0-483.99, 485-487) and by respiratory failure or sepsis, and had to meet the study definition of pneumonia prior to inclusion. Hospital admission was considered when the patient remained in the ED more than $24 \mathrm{~h}$ before discharge or was transferred to the ward or the ICU. The following cases were excluded: hospital-acquired pneumonia (diagnosed more than $48 \mathrm{~h}$ after hospital admission), witnessed aspiration pneumonia, solid organ or bone marrow transplantation, and alternative diagnoses (tuberculosis, cardiac failure or organizing pneumonia).

\section{Definitions}

Pneumonia was defined as the presence of a new alveolar opacity on chest radiography plus one or more of the following: fever (temperature $>38^{\circ} \mathrm{C}$ ) or hypothermia (temperature $<35^{\circ} \mathrm{C}$ ), cough with or without sputum production, pleuritic chest pain and altered breath sounds on auscultation.

Chronic obstructive pulmonary disease (COPD) was defined as a postbronchodilator FEV1/FVC ratio below 0.7 in accordance with the Global Initiative for Chronic Obstructive Lung Disease (GOLD) criteria in a patient with a smoking habit of more than 10 pack-years [21].

Immunocompromised group (ICP) included human immunodeficiency virus infection (HIV), neoplastic disease treated with chemotherapy or radiation therapy in the previous 3 months, hematological malignancy, anesplenia, and immunosuppressive therapy including chronic corticosteroid therapy ( $>8 \mathrm{mg} / \mathrm{d}$ methylprednisolone or equivalent for more than 30 days) and non-steroid immunosuppressive therapy.

Health Care-Associated Pneumonia (HCAP) was defined according to the criteria reported by the 2005 IDSA/ ATS Guidelines [22]. Episodes that did not meet the criteria for HCAP or ICP were classified as CAP [23].

\section{Data collection}

The following parameters were recorded in the ED: age, sex, smoking status, alcohol habits, and drug consumption, comorbidities, antibiotic treatment in the three months prior to ED visit, clinical symptoms, clinical signs, arterial blood gas measurements, chest radiograph findings, laboratory parameters, diagnostic procedures, and empiric antibiotic therapy. Previous functional status was evaluated by Barthel scale obtained through hospital databases and the Shared Medical Record of Catalonia (the CatSalut program), and the Charlson index was calculated based on data stored in the hospital database [24]. All patients were classified according to the pneumonia severity index (PSI). Intensive care unit (ICU) admission, patient treatment restrictions (do-not-resuscitate orders) and mortality were also recorded. 


\section{Microbiological evaluation}

The hospital protocol included the performance of two blood cultures, respiratory secretions (when feasible) and nasopharyngeal swabs (in suspected cases of Influenza virus in epidemic periods). Likewise, pleural fluid was analyzed in case of pleural effusion. Samples were obtained for bacterial culture before starting antibiotic therapy in the ED. Likewise, urine samples for $S$ pneumoniae and Legionella pneumophila antigen detection were routinely obtained before antibiotic therapy unless urgent antibiotic therapy was mandatory, and tested using the Binax NOW immunochromatography method (Alere BinaxNOW, Streptococcus pneumoniae Antigen Card; Alere Inc., Waltham, MA). We considered the presence of positive urine antigens as evidence of a bacterial infection. Serological tests were not used routinely to detect atypical organisms. In patients in whom microbiological results were negative, or who did not undergo microbiological tests, the etiology was considered unknown. Bronchoscopic samples such as tracheobronchial aspirate or bronchoalveolar lavage from the lower airways were obtained in intubated patients.

\section{Antimicrobial therapy}

Information was obtained on empirical antibiotic therapy given within the first $24 \mathrm{~h}$ of admission. The antibiotic regimen chosen by the attending physician was initiated in the ED in accordance with the main national reference guidelines [4]. https://www.archbronconeumol.org/es-linkresolvernormativas-el-diagnostico-el-tratamiento-13074594

Antipseudomonal $\beta$-lactam ( $\beta$-APS) was considered as any regimen that included an intravenous antipseudomonal antibiotic: carbapenems (meropenem, imipenem), antipseudomonal cephalosporin (ceftazidime, cefepime) or $\beta$-Lactam/ $\beta$-lactamase inhibitor (piperacillin-tazobactam) in monotherapy or in combination.

Antipseudomonal quinolone was defined when levofloxacin was administered in monotherapy.

Prior antibiotic therapy was defined as the exposure to antibiotics above $48 \mathrm{~h}$ during the 3 months prior to hospitalization.

Prior $P$. aeruginosa infection/colonization was defined as confirmed infection/colonization within the year prior to hospitalization, available from patients' records.

\section{Statistical analysis}

The SPSS statistical package version 21.0 (SPSS; Chicago, Illinois) was used for the statistical analysis. Results for categorical variables were expressed as absolute and relative frequencies, and continuous variables were expressed as mean values and standard deviation (SD). Differences in demographic and clinical characteristics between groups were assessed using the Chi-squared test and Fisher's exact test for categorical variables. Means were compared using ANOVA or non-parametric tests when distribution was non-normal. Variables that might have influenced the treatment decision were selected for analysis: prior antibiotic exposure, prior hospitalization, nursing home residence, comorbidities such as COPD, bronchiectasis, diabetes mellitus, chronic liver disease, chronic heart failure, and criteria of immunosuppression. Likewise, episode severity, measured by the need for ICU admission, radiological extent and urine antigen detection of S. pneumoniae were also considered.

Multivariate analysis using stepwise logistic regression was performed with prescription of empirical $\beta$-APS as a dependent variable. Variables showing a univariate association $(p<0.05)$ were included in the model as covariates. The same analysis was run for levofloxacin as dependent variable. Hosmer-Lemeshow goodness-of-fit test was used for model calibration.

Results were expressed as crude and adjusted odds ratios (OR) with $95 \%$ confidence intervals $(95 \% \mathrm{CI})$. The level of significance was set at 0.05 (two-tailed).

\section{Results \\ Cohort description}

A total of 704 consecutive episodes of pneumonia in adults (669 patients) were recorded during the study period, 549 of whom required hospitalization. Pneumonia was classified as CAP in 295/549 (53.7\%), as ICP in $125(22.8 \%)$ and as HCAP in 129 (23.5\%). The main demographic and clinical characteristics of hospitalized patients are shown in Table 1. The mean age of the population was 71.2 (SD 16.4) years; $64.9 \%$ were men, mean Charlson comorbidity index was 2.5 (SD 2.3) and mean Barthel scale 85.6 (SD 23). HCAP group had worse functional status and more treatment restrictions than the other groups, while the Charlson index and 30-day mortality were highest in the ICP group.

\section{Antibiotic regimens and microbiology}

For initial treatment in the ED, 305 (55.6\%) patients were prescribed one antibiotic and 244 (44.4\%) were prescribed a combination regimen. Eighty-five percent of prescriptions complied with the SEPAR guidelines (for the purpose of study, $\beta$-APS use was considered non-concordant). Empirical antimicrobial regimens are summarized in Table 2 . The most frequently prescribed antibiotics in monotherapy were levofloxacin in $129(42.3 \%)$ and amoxicillin-clavulanate in 108 (35.4\%). Ceftriaxone plus azithromycin (in 190, 34.2\%) was the most common combination. In 78 patients $(14 \%)$ antipseudomonal $\beta$-lactam ( $\beta$-APS) was prescribed in monotherapy or in combination. The empirical use of $\beta$-APS differed between groups and was significantly more frequent in ICP and HCAP (CAP, 6.4\%; ICP, 26.4\%; HCAP, 19.4\%; $p<0.001$ ). 
Table 1 Epidemiological and clinical characteristics of hospitalized patients

\begin{tabular}{|c|c|c|c|c|}
\hline Variable & Total $n=549$ & CAP $n=295$ & $\mathrm{ICP} n=125$ & $\operatorname{HCAP} n=129$ \\
\hline Age, mean $\pm S D$ & $71.2 \pm 16.4$ & $67.9 \pm 17.4$ & $68.8 \pm 15$ & $81.1 \pm 10.6$ \\
\hline Gender (male) & $356(64.8)$ & $181(61.4)$ & $93(74.4)$ & $82(63.6)$ \\
\hline Current/former smoker & $328(59.7)$ & $173(59.6)$ & $87(69.6)$ & $68(52.7)$ \\
\hline Charlson index, mean \pm SD & $2.5 \pm 2.3$ & $1.6 \pm 1.7$ & $4.5 \pm 2.6$ & $2.4 \pm 1.8$ \\
\hline Barthel scale, mean $\pm S D$ & $85.5 \pm 23$ & $91.4 \pm 18.1$ & $87.5 \pm 18.2$ & $70.5 \pm 29.5$ \\
\hline Diabetes mellitus & $164(29.9)$ & $83(28.1)$ & $33(26.4)$ & $48(37.2)$ \\
\hline \multicolumn{5}{|l|}{ COPD severity } \\
\hline GOLD I-III & $95(17.3)$ & $56(19.0)$ & $17(15.6)$ & $22(17.1)$ \\
\hline GOLD IV & $38(7.1)$ & $17(5.8)$ & $7(5-6)$ & $15(11.6)$ \\
\hline Bronchiectasis & $24(4.4)$ & $11(3.7)$ & $6(4.8)$ & $7(5.4)$ \\
\hline Chronic heart disease & $107(19.5)$ & 49 (16.6) & 19 (15.2) & $39(30.2)$ \\
\hline Dementia & $86(15.7)$ & $33(11.2)$ & $13(10.4)$ & $40(31)$ \\
\hline Cerebrovascular disease & $77(14)$ & 40 (13.6) & $9(7.2)$ & $28(21.7)$ \\
\hline Chronic renal failure & $81(14.8)$ & $34(11.5)$ & $17(13.6)$ & $30(23.3)$ \\
\hline Chronic liver disease & $44(8)$ & $13(4.4)$ & $23(18.4)$ & $8(6.2)$ \\
\hline \multicolumn{5}{|l|}{ PSI risk class } \\
\hline$|-|||$ & $181(33.0)$ & $141(47.8)$ & $22(17.6)$ & $18(14.0)$ \\
\hline IV & $212(38.6)$ & $103(34.9)$ & $49(39.2)$ & $60(46.5)$ \\
\hline V & $156(28.4)$ & $51(17.3)$ & $54(43.2)$ & $51(39.5)$ \\
\hline ICU admission & $59(10.7)$ & $40(13.6)$ & $16(12.8)$ & $3(2.3)$ \\
\hline 30-day- mortality & $52(9.5)$ & $15(5.1)$ & $22(17.6)$ & 15 (11.6) \\
\hline Do-not-resuscitate orders & $86(15.7)$ & $24(8.1)$ & $27(21.6)$ & $35(27.1)$ \\
\hline Prior antibiotic therapy & $167(30.4)$ & $37(12.5)$ & $48(38.4)$ & $82((63.6)$ \\
\hline Prior hospitalization & $128(23.3)$ & - & $45(36.0)$ & $83(64.3)$ \\
\hline Nursing home residence & $51(9.3)$ & - & $7(5.6)$ & $44(33.8)$ \\
\hline
\end{tabular}

SD Standard deviation, COPD Chronic obstructive pulmonary disease, GOLD Global initiative for chronic obstructive lung disease, PSI Pneumonia severity index, ICU Intensive care unit, ICP Immunocompromised group

We collected blood cultures in 340 episodes, and 145 valid respiratory specimens were retrieved. An etiological diagnosis was made in 184 patients (33.5\%): $50(40 \%)$ in the ICP group, $103(35 \%)$ in the CAP group and 31 $(24 \%)$ in the HCAP group $(p=0.02)$. Streptococcus pneumoniae was the most frequently isolated pathogen (124/ 549, 22.6\%): the pneumococcal urine antigen test was positive in 111/389 (28.5\%) of patients evaluated. Other microorganisms had a lower frequency: Haemophilus influenzae 16 (2.9\%), Moraxella catarrhalis 11 (2\%) and Enterobacteriaceae 10 (1.8\%). Pseudomonas aeruginosa was isolated in $9 / 549$ (1.6\%): three cases in the CAP group, two in the ICP group and four in the HCAP group. Moderate-severe COPD was present in five and nine patients respectively and a previous isolate of Pseudomonas aeruginosa was documented in two of them. Bacteremia occurred in only one patient, with chronic dialysis.

The distribution of microorganisms across the groups is shown in Fig. 1.
Determinants of empirical antipseudomonal use

The main factors determining selection of antipseudomonal antibiotics are summarized in Table 3. Two patterns of utilization were documented. The first was based on monotherapy with levofloxacin, which was not administered with antipseudomonal intention and was restricted to patients with low severity-of-illness. Only three patients in the ICU received quinolones; even in non-ICU patients, these agents were mainly prescribed in patients with PSI I-III and limited radiological extent. In contrast, negative pneumococcal antigen was a key factor in the prescription of levofloxacin. The other documented pattern was based on the prescription of $\beta$-APS in monotherapy (or in combination), used for antipseudomonal coverage. Site of care was the most important determinant of $\beta$-APS prescription: in fact, $\beta$-APS was the initial treatment in $22 / 59$ (35.6\%) patients in the ICU and in 56/490 (11.4\%) in ward-admitted patients $(p<$ $0.0001)$. This association was confirmed in the multivariate analysis (OR: 8.16; 95\% CI: 3.69-18.06; $p<0.0001$ ). 
Table 2 Description of initial antibiotic therapy for 549 inpatients

\begin{tabular}{|c|c|c|c|c|}
\hline Initial therapy & Total $n=549$ & CAP $n=295$ & $\mathrm{ICP} n=125$ & $\operatorname{HCAP} n=129$ \\
\hline Monotherapy & $305(55.6)$ & $156(52.9)$ & $64(51.2)$ & $85(65.9)$ \\
\hline Amoxicillin-clavulanate & $108(35.4)$ & $56(35.9)$ & $14(21.9)$ & $38(44.7)$ \\
\hline Levofloxacin & $129(42.3)$ & $76(48.7)$ & $24(37.5)$ & $29(34.1)$ \\
\hline Ceftriaxone & $22(7.2)$ & $13(8.3)$ & $7(10.9)$ & $2(2.4)$ \\
\hline$\beta$-APS & $45(14.8)$ & $11(7.1)$ & $18(28.1)$ & $16(18.8)$ \\
\hline Piperacillin-tazobactam & $26(8.5)$ & $3(1.9)$ & $12(18.8)$ & $11(12.9)$ \\
\hline Cefepime & $3(1.0)$ & $2(1.3)$ & $1(1.6)$ & $0(0.0)$ \\
\hline Meropenem & $16(5.2)$ & $6(3.8)$ & $5(7.8)$ & $5(5.9)$ \\
\hline Others & $1(0.3)$ & & & \\
\hline Combination & $244(44.4)$ & $139(47.1)$ & $61(48.8)$ & $44(34.1)$ \\
\hline Ceftriaxone + macrolide & $190(77.9)$ & $119(85.6)$ & $41(67.2)$ & $30(68.2)$ \\
\hline$\beta$-APS + quinolone or aminoglycoside or macrolide & $33(13.5)$ & $9(6.5)$ & $15(24.6)$ & $9(20.5)$ \\
\hline Others & $21(8.6)$ & $11(7.9)$ & $5(8.2)$ & $5(11.4)$ \\
\hline
\end{tabular}

$\beta$-APS Antipseudomonal $\beta$-lactam

Prior hospitalization was another significant determinant of $\beta$-APS use in community-acquired pneumonia (OR: 6.76; 95\% CI: 3.01-15.14; $p<0.0001$ ). This factor was part of the HCAP definition and was present in up to $36 \%$ of the ICP population. In addition, an association was found between $\beta$-APS prescription and severe COPD and immunosuppression (ICP population), which are classical risk factors for $P$ aeruginosa infection.

Among the 381 episodes with blood cultures and/ or respiratory samples available we tested the determinants of antibiotic use as risk factors for $P$ aeruginosa recovery. Only multilobar involvement (7/9 vs $119 / 372, p=0.007)$, prior antibiotic (6/9 vs $109 / 372$,

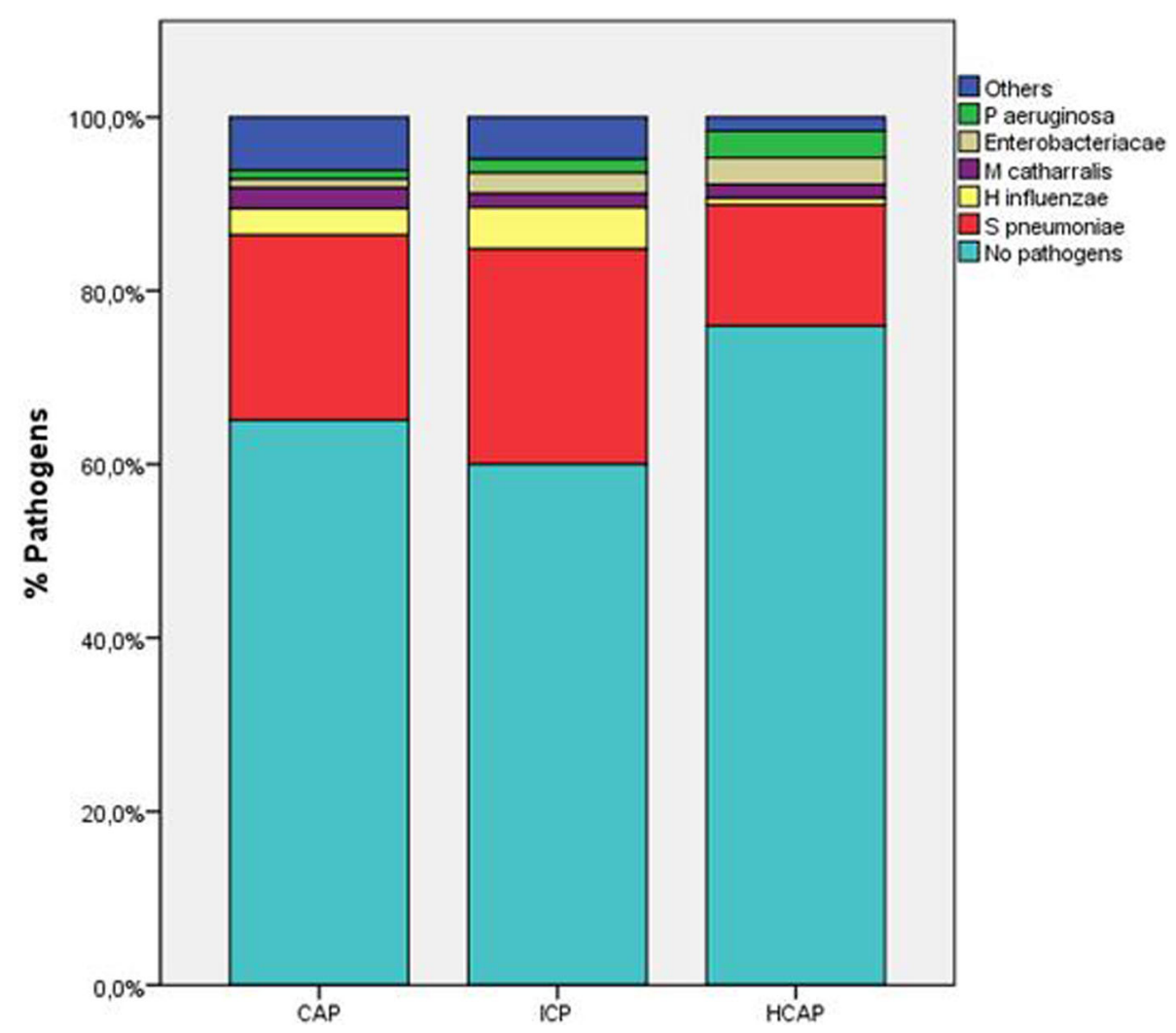

Fig. 1 Distribution of pathogens 
Table 3 Analysis of factors associated with empirical antipseudomonal use in pneumonia. Univariate and multivariate logistic regression analysis

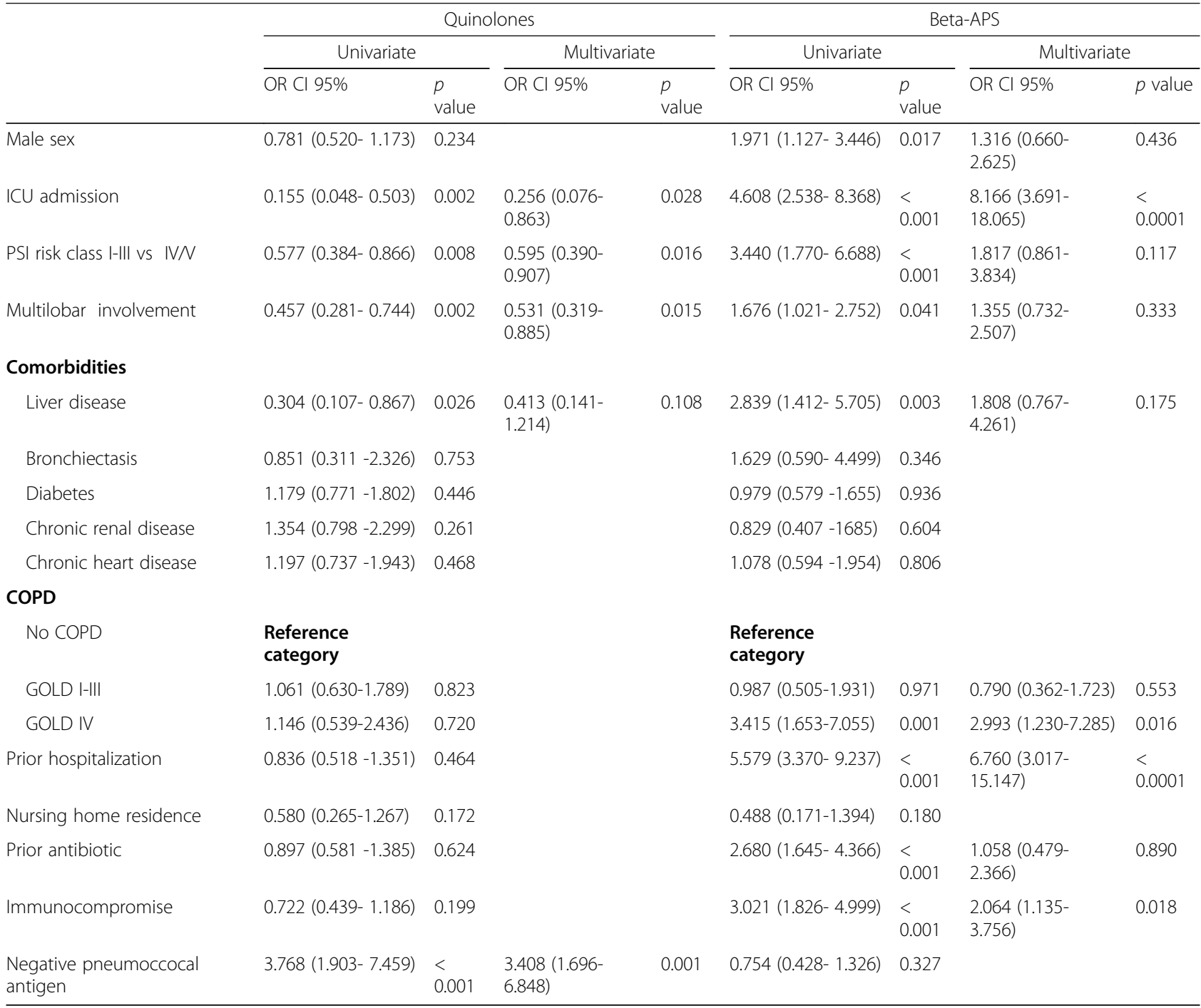

SD standard deviation, ICU intensive care unit, PSI pneumonia severity index, COPD chronic obstructive pulmonary disease, GOLD global initiative for chronic obstructive lung disease

$p=0.025)$ and severe COPD (3/9 vs 26/372, $p=$ $0.024)$ presented significant associations. When prior $P$ aeruginosa infection/colonization was included in the model, it was also associated with $P$ aeruginosa pneumonia $(2 / 9$ vs $6 / 372, p=0.013)$.

\section{Discussion}

In this study we report the drivers of antibiotic prescription for pneumonia in the ED. Nearly $37 \%$ of hospitalized patients received antibiotics with potential antipseudomonal activity; although $14 \%$ of prescriptions were intended to provide $P$ aeruginosa coverage, this pathogen was documented in only $1.6 \%$ of episodes. Severity-of-illness, identified as the need for intensive care unit admission, and prior hospitalization were the main factors in the decision to prescribe $\beta$-APS. In contrast, the prescription of levofloxacin in monotherapy was mainly associated with the presence of negative $S$ pneumoniae urine antigen and less severe disease.

Few studies carried out in the ED have focused on the determinants of antibiotic use in pneumonia, even though rates of inappropriate antibiotic treatment (for whatever reason) are known to be around 50\% [25]. Improving adherence to guidelines has been shown to raise the rates of appropriate treatment [26, 27]. In our study adherence to guidelines was high, around $85 \%$, but the etiological findings show that the use of broad spectrum antibiotics could be reduced.

Therefore, although the indication for respiratory fluoroquinolones was to provide coverage against potential S. pneumoniae strains with reduced betalactam susceptibility, Enterobacteriaceae and particularly atypical 
organisms, in agreement with the SEPAR guidelines [4], the potential of levofloxacin as an antipseudomonal antibiotic should be borne in mind.

The specific determinants of the choice of levofloxacin over other options have not been studied in depth. In our study, apart from its being restricted to ward use, the most intriguing finding was its association with unilobar pneumonia, suggesting that radiological spread is interpreted as a sign of severity, as reported elsewhere $[28,29]$. Another interesting finding was the association with negative pneumococcal urine antigen tests. The presence of a positive pneumococcal urine antigen test is indicative of $S$ pneumoniae etiology, but therapy is based on the result in fewer than $15 \%$ of cases [30]. Although outcomes with fluoroquinolones and $\beta$-lactams seem to be similar, it is less clear whether a $\beta$ lactam/ macrolide combination is superior to fluoroquinolone monotherapy; the findings are conflicting and the data in patients with severe disease are insufficient to draw conclusions [31]. Thus, attending physicians should be aware of this susceptibility pattern and should bear in mind the recent EMA warning to restrict indications of fluoroquinolones due to potentially severe adverse events, due to the increased risk of cardiac events and aneurysmal rupture in the elderly; indeed, safer treatments such as $\beta$-lactams are recommended [31].

In the case of the coverage of $P$ aeruginosa, the clinical practice guidelines range substantially, from a non-specific recommendation [3] to a recommendation in critical patients with specific risk factors $[1,4]$. In general, and in keeping with our results, overtreatment has been reported in several studies worldwide, reaching nearly $40 \%$ of patients in some cases [11, 32-34]. However, when evaluating severe episodes, the failure to cover $P$ aeruginosa is associated with increased mortality. For instance, in a multicentre study of 529 severely ill CAP admitted to the ICU, despite correct treatment according to IDSA guidelines in 15 (75\%) of 20 cases of Pseudomonas aeruginosa infection, antimicrobial treatment at admission was inadequate. Chronic obstructive pulmonary disease, malignancy, previous antibiotic exposure, and radiographic findings demonstrating rapid spread of disease were associated with $P$ aeruginosa pneumonia [9].

Few studies have evaluated aetiology according to severity-of-illness at ED presentation; Cilloniz and colleagues [35] identified Gram-negative enteric bacteria and $P$ aeruginosa as being more frequent in higher-risk groups than in low-risk groups, strengthening the argument that coverage of Gram-negative organisms is needed for these patients. However, in their study, only $5 \%$ of patients admitted to a respiratory ICU presented $P$ aeruginosa.

In our study, antipseudomonal coverage was provided in 22/59 of cases admitted to ICU, but in fact none of these patients were infected by $P$ aeruginosa.
In severe episodes of CAP requiring vasopressors or mechanical ventilation, beta-lactam/macrolide combinations were preferred in view of the evidence of reduced mortality due to the immunomodulatory effects of macrolides [36, 37]. Moreover, in agreement with recent recommendations [38] antimicrobial de-escalation is a common practice in the ICU, once the identity of organism has been confirmed by laboratory tests.

In our study, site of care was a more decisive factor than high PSI; $35.6 \%$ of ICU patients received $\beta$-APS compared with $11.4 \%$ of ward-admitted patients $(p<0.0001)$.

Another key determinant of prescription in our study was prior hospitalization. This variable is inherent to the HCAP definition [22] in an attempt to identify patients at risk of infection by multidrug-resistant microorganisms (MDR), but it has been associated with an unjustified increase in antipseudomonal prescription in patients with pneumonia, despite the lack of any increase in nosocomial pathogens [39]. Nevertheless, several recent scores attempting to refine risk factors of MDR systematically include this factor $[20,40]$. In a study performed with 935 hospitalized patients which included immunosuppressed patients, prior hospitalization was the main risk factor for MDR [20]. Although we used the traditional definition with a cut-off of $48 \mathrm{~h}$, longer hospital stay has been associated with an increased risk of MDR infection [41], a factor that was not analysed in our study.

Other factors related to the use of $\beta$-APS were immunosuppression and severity of COPD, which are well-known risk factors for $P$ aeruginosa infection. In our study severe COPD patients accounted for $60 \%$ of those infected; in the worldwide study by Restrepo et al (42) COPD was the main comorbidity, but immunosuppression was present in only two patients.

Determinants of $P$ aeruginosa coverage used in the ED were not useful in predicting $P$ aeruginosa infection in our study. However, prior $P$ aeruginosa infection/colonization together with severe COPD seem to be good predictors, although multivariate analysis was not carried out due to the low number of $P$ aeruginosa episodes. These results are in agreement with Restrepo et al [42] and with the 2019 ATS/ IDSA guidelines for community-acquired pneumonia [43] recommendations.

The most consistent risk factor for PA is prior colonization. Unfortunately, this information is not always available at the ED of a referral center because not all CAP patients have prior isolates. In any case, our data suggest that potential prior PA respiratory colonization reported in computerized clinical records should be checked in the ED.

The main limitation of this study is its single-centre retrospective design. Furthermore, the generalizability of the findings is limited as this is a single-centre cohort from 2010 to 2011 that may no longer reflect current clinical practices or pathogen distribution; for example, moxifloxacin is not approved for systemic use in Spain, a circumstance that may 
limit the generalization of our findings. However, the singlecentre design allowed us to avoid confounding factors such as differences in protocols or bias in patient selection.

\section{Conclusions}

Antipseudomonal prescription was common, in spite of the very low incidence of Pseudomonas aeruginosa. Levofloxacin was prescribed to patients with low severity and negative pneumococcal urine antigen tests. Antibiotic prescription intended to provide $P$ aeruginosa coverage was guided mainly by severity-of-illness, prior hospitalization and, less frequently, by specific risk factors. However, these factors were not associated with $P$ aeruginosa episodes. Only prior $P$ aeruginosa infection/ colonization and severe COPD seem to be reliable in clinical practice.

\section{Abbreviations}

ATS: American Thoracic Society; CAP: Community-acquired pneumonia; HCAP: Healthcare-associated pneumonia; ICP: Immunocompromise; IDSA: Infectious Diseases Society of America; COPD: Chronic obstructive pulmonary disease; GOLD: Global initiative for chronic obstructive disease; PSI: Pneumonia severity index; EMA: European Medicines Agency

\section{Acknowledgments}

We thank Michael Maudsley for providing an outline for this manuscript and for support in editing and journal styling.

\section{Authors' contributions}

$N A, M G, J F, J V, A M, E M$ and JR jointly designed the study. NA and MG reviewed each patient's chart with the hospital's program for storing digital images (RAIM Server) to confirm the diagnosis of pneumonia based on clinical presentation and chest radiograph. NA, JF and AM collected the data. MG and JR performed the statistical analyses. NA drafted the manuscript and all authors contributed to the final manuscript. The authors read and approved the final version of the manuscript.

\section{Funding}

Supported in part by Centro de Investigacion Biomedica en Red (CIBERES), Instituto de Salud Carlos III, Madrid, Spain. The funding agency had no role in the study design; collection, analysis, or interpretation of data; drafting of the manuscript; or decision to submit for publication.

\section{Availability of data and materials}

The datasets used in this study are available from the corresponding author upon reasonable request.

\section{Ethics approval and consent to participate}

The study protocol was approved by the Hospital Ethics Committee on Human Research (No. 2014/538). The need for informed consent was waived because patient information was anonymized and de-identified prior to analysis.

\section{Consent for publication}

Not applicable.

\section{Competing interests}

JR has received research grants and consulting fees from BAYER and PARATEK. The pharmaceutical industry did not contribute to the funding of this study. The other authors declare that they have no competing interests.

\section{Author details}

'Department of Respiratory Medicine, Parc Taulí Hospital Universitari, Institut d'Investigació i Innovació Parc Taulí I3PT, Universitat Autònoma de Barcelona, Sabadell, Spain. ${ }^{2}$ CIBER de Enfermedades Respiratorias (CIBERES), Instituto de Salud Carlos III, Madrid, Spain. ${ }^{3}$ Emergency Department, Parc Taulí Hospital Universitari, Institut d'Investigació i Innovació Parc Taulí I3PT, Universitat
Autònoma de Barcelona, Sabadell, Spain. ${ }^{4}$ Critical Care Center, Parc Taulí Hospital Universitari, Institut d'Investigació i Innovació Parc Taulí I3PT, Universitat Autònoma de Barcelona, Sabadell, Spain. ${ }^{5}$ Department of Pharmacy, Parc Taulí Hospital Universitari, Institut d'Investigació i Innovació Parc Taulí I3PT, Universitat Autònoma de Barcelona, Sabadell, Spain. ${ }^{6}$ Vall d'Hebron Institute of Research (VHIR), Barcelona, Spain. ${ }^{7}$ Research Department, CHU Nîmes, Université Montpellier-Nîmes, Nîmes, France.

Received: 17 November 2019 Accepted: 13 March 2020

Published online: 03 April 2020

\section{References}

1. Mandell LA, Wunderink RG, Anzueto A, Bartlett JG, Campbell GD, Dean NC, et al. Infectious Diseases Society of America/American Thoracic Society consensus quidelines on the management of community-acquired pneumonia in adults. Clin Infect Dis. 2007:44:S27e72.

2. Woodhead M, Blasi F, Ewig S, Garau J, Huchon G, Leven M, et al. Guidelines for the management of adult lower respiratory tract infections full version. Clin Microbiol Infect. 2011;17:E1e59.

3. Lim WS, Baudouin SV, George RC, Hill AT, Jamieson C, Le Jeune I, et al. BTS guidelines for the management of community acquired pneumonia in adults: update 2009. Thorax. 2009;64:iii 1 e55.

4. Alfageme I, Aspa J, Bello S, Blanquer J, Blanquer R, Borderías L, et al. Guidelines for the diagnosis and management of community-acquired pneumonia. Spanish Society of Pulmonology and Thoracic Surgery (SEPAR). Arch Bronconeumol. 2005:41(5):272-89.

5. Prina E, Ranzani OT, Torres A. Community-acquired pneumonia. Lancet. 2015;386:1097-108.

6. Musher DM, Thorner AR. Community-acquired pneumonia. N Engl J Med. 2014:371:1619-28.

7. Reyes Calzada S, Martinez Tomas R, Cremades Romero MJ, Martinez Moragon E, Soler Cataluna JJ, Menendez Villanueva R. Empiric treatment in hospitalized community-acquired pneumonia. Impact on mortality, length of stay and re-admission. Respir Med. 2007;101:1909-15.

8. Kollef MH, Micek ST. Patients hospitalized with pneumonia: determining the need for broad-spectrum antibiotic therapy. Clin Infect Dis. 2012;54(4):479-82.

9. Bodí M, Rodríguez A, Solé-Violán J, Gilavert MC, Garnacho J, Blanquer J, et al. Community-acquired pneumonia intensive care units (CAPUCI) study investigators. Antibiotic prescription for community-acquired pneumonia in the intensive care unit: impact of adherence to Infectious Diseases Society of America guidelines on survival. Clin Infect Dis. 2005:41(12):1709-16.

10. Singanayagam A, Chalmers JD. Severity assessment scores to guide empirical use of antibiotics in community acquired pneumonia. Lancet Respir Med. 2013;1(8):653-62.

11. Webb BJ, Sorensen J, Jephson A, Mecham I, Dean NC. Broad-spectrum antibiotic use and poor outcomes in community-onset pneumonia: a cohort study. Eur Respir J. 2019;54(1):1900057.

12. Respipedia. Respiratory infections. Pseudomonas aeruginosa infections. Www.Ers-education.Org/home/browse-all-content.Aspx?!dparent=207389. Date last Accessed: 31 Jan 2019.

13. Arancibia F, Bauer TT, Ewig S, Mensa J, Gonzalez J, Niederman MS, et al. Community-acquired pneumonia due to gram-negative bacteria and Pseudomonas aeruginosa: incidence, risk, and prognosis. Arch Intern Med. 2002;162(16):1849-58.

14. Kang $\mathrm{Cl}$, Song JH, Oh WS, Ko KS, Chung DR. Peck KR; Asian network for surveillance of resistant pathogens (ANSORP) study group. Clinical outcomes and risk factors of community-acquired pneumonia caused by gram-negative bacilli. Eur J Clin Microbiol Infect Dis. 2008;27(8):657-61.

15. von Baum H, Welte T, Marre R, Suttorp N, Ewig S, CAPNEIZ study group. Community-acquired pneumonia through Enterobacteriaceae and Pseudomonas aeruginosa: diagnosis, incidence and predictors. Eur Respir J. 2010;35(3):598-605.

16. Falguera M, Carratala J, Ruiz-Gonzalez A, Garcia-Vidal C, Gazquez I, Dorca J, et al. Risk factors and outcome of community-acquired pneumonia due to gram-negative bacilli. Respirology. 2009;14:105-11.

17. Sibila O, Laserna E, Maselli DJ, Fernandez JF, Mortensen EM, Anzueto A, et al. Risk factors and antibiotic therapy in $P$ aeruginosa communityacquired pneumonia. Respirology. 2015;20:660-6.

18. Cilloniz C, Gabarrus A, Ferrer M, Puig de la Bellacasa J, Rinaudo M, Mensa J, et al. Community-acquired pneumonia due to multidrug- and nonmultidrug-resistant pseudomonas aeruginosa. Chest. 2016;150:415-25.

19. Niederman MS, Mandell LA, Anzueto A, Bass JB, Broughton WA, Campbell $G D$, et al. American Thoracic Society. Guidelines for the management of 
adults with community-acquired pneumonia. Diagnosis, assessment of severity, antimicrobial therapy, and prevention. Am J Respir Crit Care Med. 2001;163(7):1730-54.

20. Aliberti S, Di Pasquale M, Zanaboni AM, Cosentini R, Brambilla AM, Seghezzi $\mathrm{S}$, et al. Stratifying risk factors for multidrug-resistant pathogens in hospitalized patients coming from the community with pneumonia. Clin Infect Dis. 2012;54(4):470-8.

21. Rabe KF, Hurd S, Anzueto A, Barnes PJ, Buist SA, Calverley P, et al. Global initiative for chronic obstructive lung disease. Global strategy for the diagnosis, management, and prevention of chronic obstructive pulmonary disease: GOLD executive summary. Am J Respir Crit Care Med. 2007;176(6):532-55.

22. American Thoracic Society; Infectious Diseases Society of America. Guidelines for the management of adults with hospital-acquired, ventilatorassociated, and healthcare-associated pneumonia. Am J Respir Crit Care Med. 2005;171(4):388-416.

23. Vallés J, Martin-Loeches I, Torres A, Diaz E, Seijas I, López MJ, et al. Epidemiology, antibiotic therapy and clinical outcomes of healthcareassociated pneumonia in critically ill patients: a Spanish cohort study. Intensive Care Med. 2014:40(4):572-81.

24. Charlson ME, Pompei P, Ales KL, MacKenzie CR. A new method of classifying prognostic comorbidity in longitudinal studies: development and validation. J Chronic Dis. 1987;40(5):373-83.

25. Oltra Hostalet F, Núñez-Núñez M, Portillo Cano MDM, Navarro Bustos C, RodríguezBaño J, Retamar Gentil P. Analysis of quality antimicrobial agent use in the emergency department of a tertiary care hospital. Emergencias. 2018;30(5):297-302.

26. Pifarre R, Falguera M, Vicente-de-Vera C, Nogues A. Characteristics of community-acquired pneumonia in patients with chronic obstructive pulmonary disease. Respir Med. 2007;101(10):2139-44.

27. Faine BA, Mohr N, Dietrich J, Meadow L, Harland KK, Chrischilles E. Antimicrobial therapy for pneumonia in the emergency department: the impact of clinical pharmacists on appropriateness. West J Emerg Med. 2017; 18(5):856-63. https://doi.org/10.5811/westjem.2017.5.33901.

28. Liapikou A, Cillóniz C, Gabarrús A, Amaro R, De la Bellacasa JP, Mensa J, et al. Multilobar bilateral and unilateral chest radiograph involvement: implications for prognosis in hospitalised community-acquired pneumonia. Eur Respir J. 2016;48(1):257-61.

29. Lisboa T, Blot S, Waterer GW, Canalis E, de Mendoza D, Rodriguez A, et al. Radiologic progression of pulmonary infiltrates predicts a worse prognosis in severe community-acquired pneumonia than bacteremia. Chest. 2009;135(1):165-72.

30. West DM, McCauley LM, Sorensen JS, Jephson AR, Dean NC. Pneumococcal urinary antigen test use in diagnosis and treatment of pneumonia in seven Utah hospitals. ERJ Open Res. 2016:19:2(4).

31. Wunderink RG, Waterer G. Advances in the causes and management of community acquired pneumonia in adults. BMJ. 2017;358:2471.

32. Jones BE, Brown KA, Jones MM, Huttner BD, Greene T, Sauer BC, et al. Variation in empiric coverage versus detection of methicillin-resistant Staphylococcus aureus and Pseudomonas aeruginosa in hospitalizations for community-onset pneumonia across 128 US veterans affairs medical centers. Infect Control Hosp Epidemiol. 2017;38(8):937-44.

33. Chalmers JD, Rother C, Salih W, Ewig S. Healthcare-associated pneumonia does not accurately identify potentially resistant pathogens: a systematic review and meta-analysispublished correction appears in. Clin Infect Dis. 2015:60(7):1143.

34. Metersky ML, Frei CR, Mortensen EM. Predictors of pseudomonas and methicillin-resistant Staphylococcus aureus in hospitalized patients with healthcare-associated pneumonia. Respirology. 2016;21(1):157-63.

35. Cilloniz C, Ewig S, Polverino E, Marcos MA, Esquinas C, Gabarrus A, et al. Microbial aetiology of community-acquired pneumonia and its relation to severity. Thorax. 2011;66:340-6.

36. Martin-Loeches I, Lisboa T, Rodriguez A, Putensen C, Annane D, GarnachoMontero J, et al. Combination antibiotic therapy with macrolides improves survival in intubated patients with community-acquired pneumonia. Intensive Care Med. 2010;36(4):612-20.

37. Restrepo MI, Mortensen EM, Waterer GW, Wunderink RG, Coalson Jر J, Anzueto A. Impact of macrolide therapy on mortality for patients with severe sepsis due to pneumonia. Eur Respir J. 2009;33(1):153-9.

38. Tabah A, Bassetti M, Kollef MH, Zahar JR, Paiva JA, Timsit JF, et al. Antimicrobial de-escalation in critically ill patients: a position statement from a task force of the European Society of Intensive Care Medicine (ESICM) and European Society of Clinical Microbiology and Infectious Diseases (ESCMID) critically ill patients study group (ESGCIP). Intensive Care Med. 2019;28:1-21.
39. Jones BE, Jones MM, Huttner B, Stoddard G, Brown KA, Stevens W, et al. Trends in antibiotic use and nosocomial pathogens in hospitalized veterans with pneumonia at 128 medical centers, 2006-2010. Clin Infect Dis. 2015;61(9):1403-10.

40. Prina E, Ranzani OT, Polverino E, Cillóniz C, Ferrer M, Fernandez L, et al. Risk factors associated with potentially antibiotic-resistant pathogens in community-acquired pneumonia. Ann Am Thorac Soc. 2015;12(2):153-60.

41. Gross AE, Van Schooneveld TC, Olsen KM, Rupp ME, Bui TH, Forsung E, et al. Epidemiology and predictors of multidrug-resistant community-acquired and health care-associated pneumonia. Antimicrob Agents Chemother. 2014;58(9):5262-8.

42. Restrepo MI, Babu BL, Reyes LF, Chalmers JD, Soni NJ, Sibila O, et al. Burden and risk factors for Pseudomonas aeruginosa community-acquired pneumonia: a multinational point prevalence study of hospitalised patients. Eur Respir J. 2018;52:1701190.

43. Metlay JP, Waterer GW, Long AC, Anzueto A, Brozek J, Crothers K, et al. Diagnosis and treatment of adults with community-acquired pneumonia. An official clinical practice guideline of the American Thoracic Society and Infectious Diseases Society of America. Am J Respir Crit Care Med. 2019;200(7):e45-67.

\section{Publisher's Note}

Springer Nature remains neutral with regard to jurisdictional claims in published maps and institutional affiliations.
Ready to submit your research? Choose BMC and benefit from:

- fast, convenient online submission

- thorough peer review by experienced researchers in your field

- rapid publication on acceptance

- support for research data, including large and complex data types

- gold Open Access which fosters wider collaboration and increased citations

- maximum visibility for your research: over $100 \mathrm{M}$ website views per year

At BMC, research is always in progress.

Learn more biomedcentral.com/submissions 